\title{
Stockage des spermatozoïdes dans l'oviducte chez les oiseaux : approche morphologique, histologique et fonctionnelle
}

\author{
JP Brillard \\ INRA, Station de recherches avicoles, I 37380 Nouzilly, France
}

(Reçu le 13 juin 1989; accepté le 23 janvier 1990)

\begin{abstract}
Résumé - Cet article est une synthèse de la bibliographie consacrée aux aspects anatomique, histologique et fonctionnel du stockage des spermatozoïdes dans l'oviducte des oiseaux domestiques (poule, dinde). Bien que des lipides ou des mucopolysaccharides soient retrouvés dans le cytoplasme des cellules composant les glandes utérovaginales (site de résidence des spermatozoïdes dans l'oviducte après insémination intravaginale), l'absence d'organites impliqués dans un cycle sécrétoire de protéines pose la question de leur synthèse possible par ces glandes. En l'absence d'un tel cycle, les microvillosités bordant la lumière des glandes pourraient être impliquées dans un processus de purification du micro-environnement des spermatozoïdes et permettre ainsi leur survie prolongée dans ce milieu. Plusieurs observations récentes laissent penser que le mécanisme qui contrôle l'entrée et/ou le stockage des spermatozoïdes dans les glandes utéro-vaginales est de nature immunodépendante et qu'il est en liaison étroite avec l'intégrité fonctionnelle du glycocalyx qui entoure les spermatozoïdes. Une étude quantitative de la vitesse de stockage avec ou sans altération de ce glycocalyx en préciserait peut-être le rôle (reconnaissance des spermatozoïdes par les glandes ou bien leur agglutination dans celles-ci) et pourrait contribuer à mieux faire connaître la nature du stockage prolongé des spermatozoïdes dans les glandes utéro-vaginales des oiseaux.
\end{abstract}

spermatozoïde / oviducte / stockage in vivo / oiseau

Summary - Oviducal storage of spermatozoa in birds : a morphological, histological and functional approach. This paper reviews the studies devoted to the anatomical, histological and functional aspects of sperm storage in the oviduct of domestic birds (hen, turkey). Despite the presence of substances, such as lipids and mucopolysaccharides, in the cytoplasm of the cells encompassing the utero-vaginal glands (site of residence of spermatozoa in the oviduct after intra-vaginal inseminations), the absence of organelles involved in protein synthesis questions the existence of a secretory cycle in these glands. In the absence of such a cycle, the microvilli bordering the lumen of the utero-vaginal glands could participate in the purification of this micro-environment, thus allowing prolonged survival of spermatozoa in this region. Recent observations indicate that the mechanism controlling the penetration and/or storage of spermatozoa in the utero-vaginal glands is of an immuno-dependent nature and is closely related to the functional integrity of the glycocalyx surrounding the spermatozoon. A quantitative study of the kinetics of the storage of spermatozoa with or without alteration of their glycocalyx might help to explain its role (recognition of spermatozoa by the uterovaginal glands or agglutination of spermatozoa within these glands). This type of experiment might also contribute to understanding the mechanism underlying the prolonged storage of spermatozoa in the utero-vaginal glands of birds. 


\section{INTRODUCTION}

Chez les vertébrés, le maintien du pouvoir fécondant des spermatozoïdes dans les voies génitales femelles varie selon les espèces. En règle générale, sa durée chez les poïkilothermes est supérieure à celle observée chez les homéothermes (Thibault et Levasseur, 1973). Chez les Mammifères, par exemple, et à de rares exceptions près, comme la chauve-souris et le lièvre (Wimsatt, 1942; Martinet et Raynaud, 1972), l'accouplement ou l'insémination artificielle doivent être réalisés dans les heures qui précèdent l'ovulation pour être efficaces. Chez la plupart des oiseaux, en revanche, le maintien du pouvoir fécondant des spermatozoïdes dans l'oviducte dépasse largement une semaine : caille, 11-12 j (Sittmann et Abplanalp, 1965); cane, 13-17 j selon les races (Fronda et al, 1940; Elder et Weller, 1954); oie, 12-17 j (Johnson, 1954; Kinney et Burger, 1960); pintade, 17-21 j (Thibault et Levasseur, 1973); poule, 3-4 sem (Nicolaïdes, 1934; Romanoff, 1960; Lake, 1967); dinde, 8-9 sem (Lorenz, 1950; McCartney, 1951; Hale, 1955). Mais les "périodes fertiles" que nous rapportons ici sont des valeurs extrêmes qui ne préjugent en rien des possibilités de chaque œuf à être fécondé et à donner naissance à un embryon viable. En fait, plus la durée qui sépare le jour de l'insémination du jour de ponte est longue, moins les chances de fécondation de l'œuf sont grandes et plus le risque de mortalité embryonnaire est élevé (Lodge et al, 1971).

Ceci pose le problème, encore mal connu, de l'efficacité du stockage in vivo des spermatozoïdes, ainsi que celui des échanges métaboliques qui favorisent leur survie dans un milieu ambiant par nature perturbé en raison des sécrétions et des mouvements multiples qui accompagnent la formation de chaque œuf. Le but de cet article est de mettre à jour les connaissances sur ce sujet en complément de plusieurs revues bibliographiques importantes (Lake, 1975; Sauveur, 1979; Bakst, 1987; Zavalet et Ogasawara, 1987).

\section{SITES DE STOCKAGE DES SPERMATO- ZOÏDES CHEZ LES OISEAUX}

\section{Stockages infundibulaire et utéro-vaginal}

C'est sans doute à Tauber (1875) et Tascher (1876), cités par Van Krey (1965) que reviennent la découverte d'un premier site de stockage des spermatozoïdes dans l'oviducte des oiseaux. Leur travail, conduit chez la poule (Gallus gallus) signalait l'existence d'une zone «alvéolaire» confinée à la partie antérieure de l'infundibulum (pavillon). Ces mêmes auteurs précisèrent que l'apparition et la disparition des spermatozoïdes dans cette zone correspondaient à l'apparition et à la disparition d'œufs fécondés dans l'oviducte. Bien plus tard, Van Drimmelen (1946) entreprit d'étudier précisément les conditions de stockage des spermatozoïdes dans l'oviducte de poules en analysant des sections d'oviductes prélevés 8 jours après insémination intrapéritonéale. Dans ces conditions, il observa de nombreux spermatozoïdes rassemblés dans les cryptes de l'infundibulum qu'il baptisa "sperm nests". Chez d'autres femelles placées en reproduction naturelle, le même auteur retrouva occasionnellement quelques spermatozoïdes dans ces cryptes et en conclut que cette zone de l'oviducte était leur site normal de résidence après insémination en accouplement. Mais Fujii (1963), ainsi que Fujii et Tamura (1963) localisèrent des glandes de forme identique à celles de l'infundibulum dans la partie utéro-vaginale de l'oviducte 
de poule; ils les nommèrent "vaginal glands". Ces glandes, appelées encore "sperm host glands" (Takeda, 1964) ou "sperm storage tubules» (Mero, Ogasawara, 1970) sont réparties dans une bande circulaire étroite $(5-10 \mathrm{~mm}$ chez la poule, 5-45 mm chez la dinde) de la muqueuse qui sépare la zone utérine de l'entrée du vagin. Dans cette zone, la muqueuse est tapissée de replis longitudinaux ayant un aspect intermédiaire entre les replis utérins et les replis vaginaux (fig 1); elle correspond à la ujonction utéro-vaginale" telle qu'elle a été définie par Bobr et al (1964). Les replis de la paroi interne de l'oviducte sont, à cet endroit, parsemés d'invaginations tubulaires (fig 2), connues le plus souvent sous le nom de "glandes utérovaginales". Ces glandes disparaissent à l'entrée du vagin, alors que la partie basale de l'utérus contient de nombreuses glandes tubulaires apparemment semblables à celles de la jonction utérovaginale, mais qui ne contiennent jamais de spermatozoïdes (Bobr et al, 1964).

En dehors de l'espèce Gallus gallus, les glandes utéro-vaginales ont été observées chez de nombreux autres oiseaux tels la dinde (Verma et Cherms, 1964), le merle à collerette (Bray et al, 1975), le canard domestique (Pal, 1977), la caille (Frieb et al, 1978), le pétrel (Hatch, 1983) et le faucon américain (Bakst et Bird, 1987). Leur présence semble donc constante et leur histologie, ainsi que leur fonctionnement, étudiés surtout chez la poule et la dinde, sont comparables. Fujii et Tamura (1963), étudiant la poule, notèrent que ces glandes se remplissaient de spermatozoïdes après insémination intravaginale et en conclurent qu'elles constituaient leur site normal de résidence après insémination par les voies naturelles. Ils assimilèrent la région glandulaire de l'infundibulum décrite par Van Drimmelen (1946) à un lieu de mise en attente provisoire des spermatozoïdes lors de leur déplacement vers l'infundibulum, site de la fécondation. Cette hypothèse fut reprise par Bobr et al (1964) et par Takeda (1964), chez la poule, puis par Prasad (1967), chez la poule et la dinde. A ce jour, les rôles relatifs des stockages infundibulaire et utéro-vaginal ne sont pas définitivement élucidés. Van Krey et al (1964) ont établi chez la poule que la fertilité persistait plus longtemps après insémination intramagnale qu'après insémination intravaginale ou intra-utérine, en dépit d'une quasi absence de stockage au niveau de la jonction utéro-vaginale. Schindler et al (1967) ont rapporté que les glandes infundibulaires sont capables de stocker des spermatozoïdes au moins aussi longtemps que les glandes utéro-vaginales et une étude conduite par Degen et Hawes (1972) révèle que le stockage peut, dans certains cas, avoir lieu dans la région infundibulaire. La méthode utilisée par ces 2 derniers auteurs consiste à retirer chirurgicalement la jonction utéro-vaginale de poules déjà en ponte ou bien juste avant celle-ci; dans ces conditions, un peu plus de la moitié des femelles opérées (principalement les plus jeunes) se remettent à pondre dans les 3 mois suivant l'intervention. Chez des poules ainsi préparées, le taux de fécondation des œufs augmente progressivement jusqu'à atteindre celui des animaux témoins, mais la mortalité embryonnaire reste très élevée $(33,6 \%$ contre $18 \%$ dans le lot témoin).

Plusieurs des travaux que nous venons d'évoquer montrent que la zone infundibulaire peut-être utilisée pour le stockage des spermatozoïdes. II n'en reste pas moins que ceux-ci n'y sont retrouvés en grand nombre qu'après l'utilisation de techniques d'études particulières et que, dans tous les cas, la mortalité embryonnaire enregistrée chez les œuts de poules ainsi traitées est supérieure à celle observée chez les poules inséminées par les voies naturelles. 

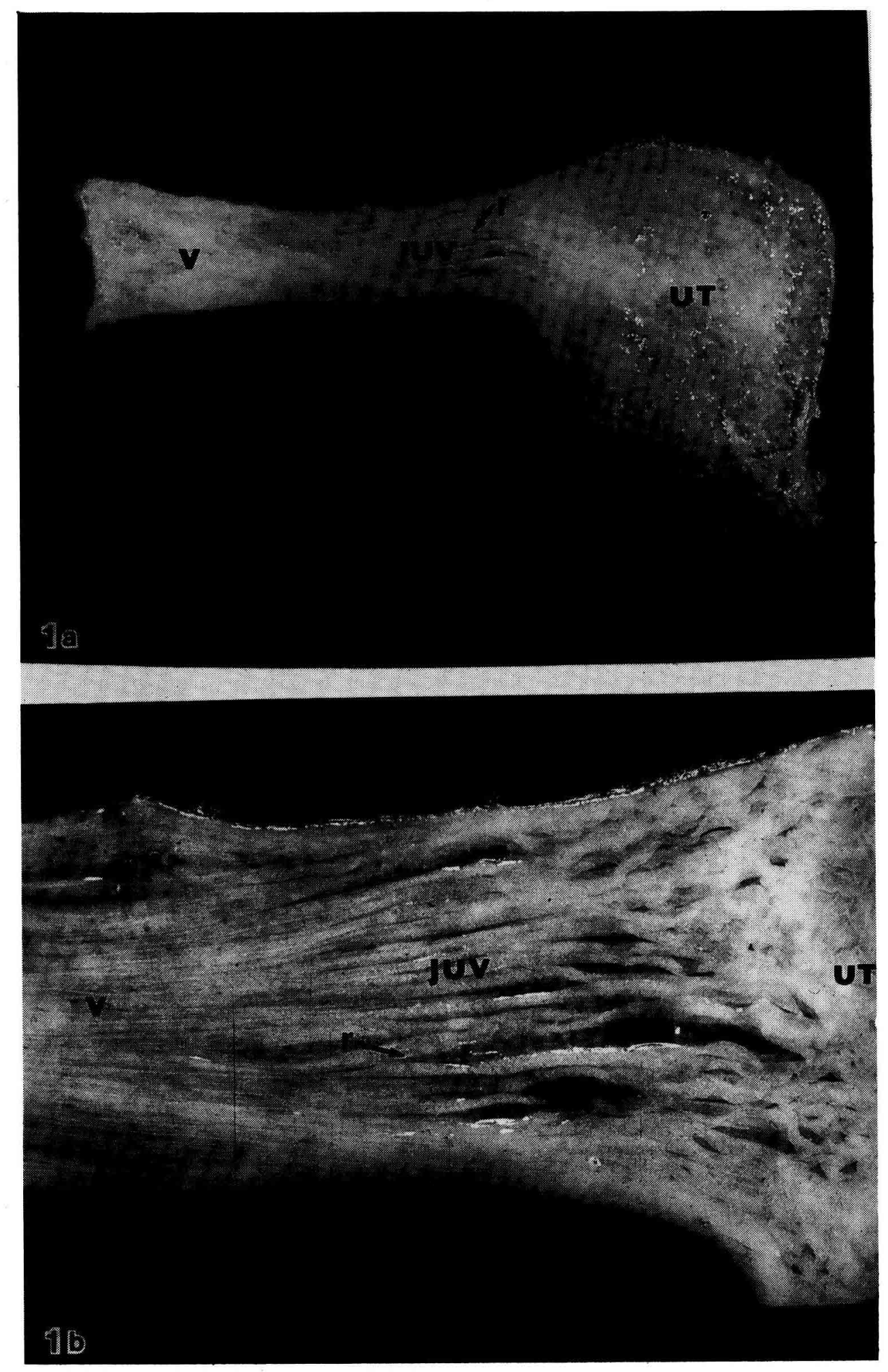
Cette augmentation de la mortalité embryonnaire chez des femelles inséminées dans la partie supérieure de l'oviducte (audessus de la jonction utéro-vaginale) reste de nature inconnue. Pour l'expliquer, Zavaleta et Ogasawara (1987) proposent 2 hypothèses qui n'ont pas, à ce jour, été vérifiées : la première mettrait en cause une augmentation des taux de fécondation polyspermique en liaison avec la présence de très nombreux spermatozoïdes sur le site de fécondation (infundibulum) lorsque le stockage infudibulaire est favorisé. La seconde hypothèse évoque ia possibilité de fécondation provoquée par des spermatozoïdes en état de dégénérescence. Cette seconde hypothèse est fondée sur les observations de Bobr et al (1964) selon lesquelles les spermatozoïdes dégénèrent rapidement après leur stockage dans l'infundibulum : elle nous paraît cependant peu vraisemblable, car on voit mal comment des spermatozoïdes stockés à proximité immédiate du lieu de fécondation vieilliraient plus rapidement que ceux normalement stockés dans la jonction utérovaginale, alors que la persistance de fertilité est, dans le premier cas, plus grande que dans le second (Van Krey et al, 1964).

La jonction utéro-vaginale doit donc être considérée comme le lieu de stockage normal des spermatozoïdes après fécondation naturelle ou insémination intravaginale (Bobr et al, 1964; Ogasawara et Fuqua, 1972). Cette jonction assure en outre une sélection des spermatozoïdes, comme nous allons le préciser et comme le suggère l'expérience de Degen et Hawes (1972).

\section{TRI DES SPERMATOZOIDES STOCKÉS DANS LA JONCTION UTÉRO-VAGINALE}

Des spermatozoïdes déposés directement dans l'utérus atteignent l'infundibulum en plus grand nombre que ceux placés à l'entrée du vagin (Allen et Grigg, 1957). Ce résultat pourrait s'expliquer par le fait qu'une partie seulement des gamètes mâles possède une mobilité suffisante pour atteindre les glandes de la jonction utéro-vaginale. En effet, ces auteurs, puis Saeki et al (1963) signalent que des spermatozoïdes morts inséminés par voie intravaginale ne parviennent pas à franchir l'utérus, alors qu'ils atteignent l'infundibulum en aussi grand nombre que des spermatozoïdes vivants, lorsqu'ils sont placés dans le magnum. Ce rôle de filtre de la région utérovaginale est confirmé par le fait que la fertilité de femelles inséminées par voie intravaginale avec du sperme de mauvaise qualité (pourcentages de spermatozoïdes morts ou anormaux élevés, faible motilité) reste faible alors qu'elle devient grande chez les femelles inséminées avec le même sperme par voie intra-utérine (Ogasawara et al, 1966). Malheureusement, cette expérience ne comporte pas d'étude sur l'éclosivité des œuts ainsi fécondés. A l'inverse, du sperme de bonne qualité inséminé par voie intravaginale conduit à des hauts niveaux de fertilité et d'éclosivité alors que, dans les mêmes conditions, l'éclosivité - mais non la fertilité - est moindre si le sperme est placé dans le magnum ou l'infundibulum (Lorenz et Ogasa-

Fig 1. a) Au plan anatomique, la jonction utéro-vaginale (JUV) est caractérisée par la présence de replis ( $r$ ) longitudinaux (de 18 à 25 en tout chez la dinde) nettement plus épais que ceux observés dans le vagin (V). Ces replis disparaissent dans la partie de jonction en contact avec l'utérus (UT); $x$ 0,35. b) Les glandes utéro-vaginales sont disséminées de part et d'autre des replis mais sont quasi absentes du tissu conjonctif intermédiaire. Noter le changement d'orientation des replis en contact avec la région utérine; $x 1$ (avec l'aimable autorisation du Dr M Bakst). 

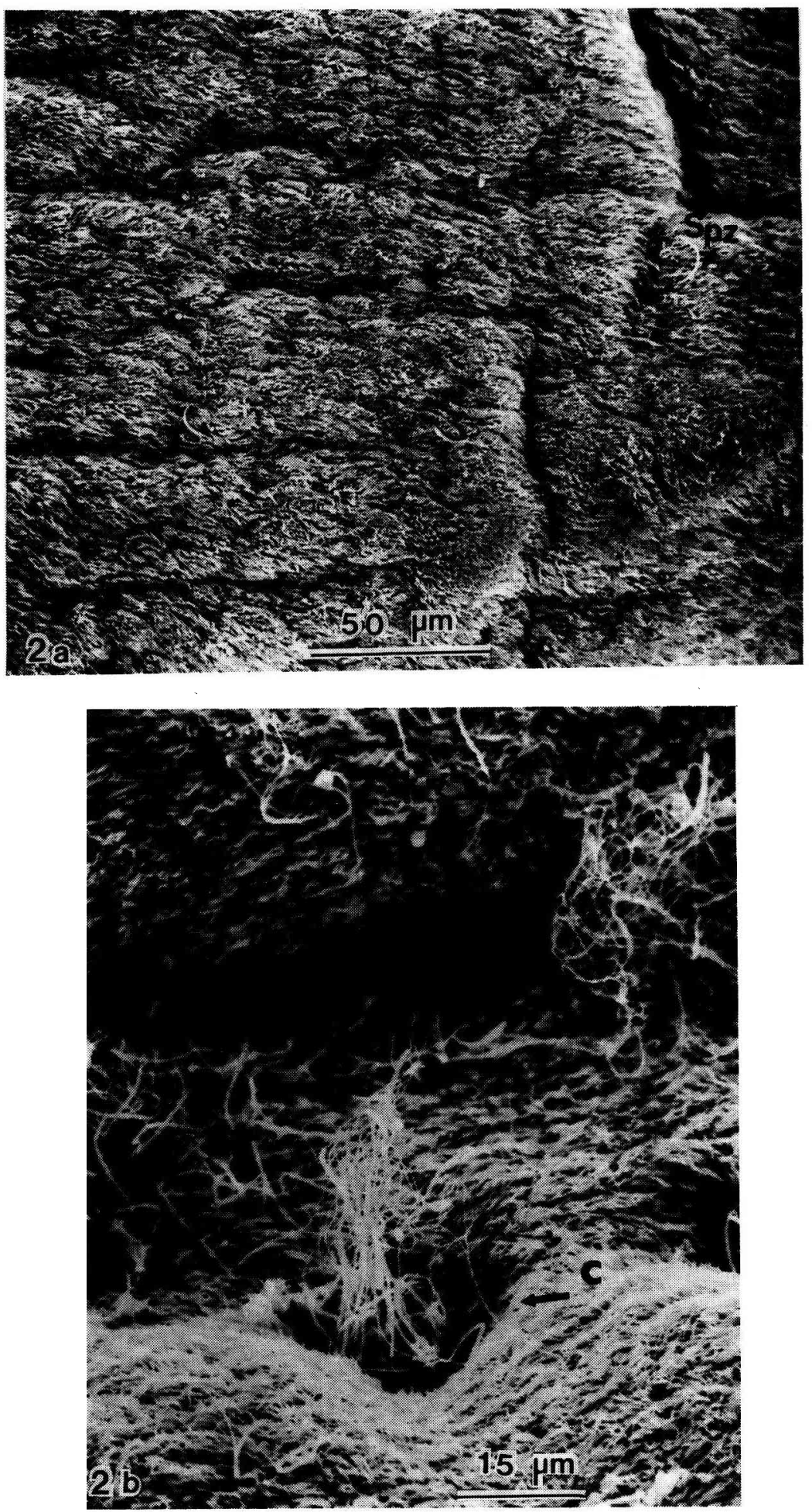
wara, 1968). L'expérience précédente indique qu'une fraction importante des spermatozoïdes présentant des altérations morphologiques ou fonctionnelles susceptibles d'entraîner la mort des embryons ne franchit jamais la jonction utérovaginale dans des conditions normales de fécondation. Ceci est indirectement confirmé par Van Krey et al (1981) qui notent que : "les études ultra-structurales des glandes utéro-vaginales ne mentionnent nulle part l'existence de spermatozoïdes en état de dégénérescence". II semble que cette capacité de tri soit d'ailleurs limitée à la jonction utéro-vaginale car des figures de dégénérescence des spermatozoïdes stockés dans les glandes infundibulaires ont été nettement identifiées par Koyanagi et Nishiyama (1981).

\section{HISTOLOGIE ET HISTOCHIMIE DES GLANDES UTÉRO-VAGINALES ET INFUNDIBULAIRES}

\section{Histologie}

Les premières descriptions histologiques de glandes infundibulaires et utérovaginales reviennent à Richardson (1935) qui les trouva «identiques entre elles", une observation confirmée par Bobr et al (1964) qui ne purent déceler d'autre différence que celle due à leur site d'implantation dans l'oviducte. Les travaux auxquels nous faisons référence ci-après portent, pour l'essentiel, sur l'étude des glandes utéro-vaginales, qui ont été, de très loin, les plus étudiées. Chez la jeune femelle impubère, ces glandes apparaissent sous la forme de simples invaginations de l'épithélium; elles sont constituées de cellules cuboïdes qui s'allongent pendant la puberté, et qui prennent, chez l'adulte, une forme columnaire (Gilbert et al, 1968). Leur développement semble être sous dépendance hormonale, car l'injection de diéthyl-stilbestrol à de jeunes poulettes de 6 semaines, c'est-à-dire bien avant l'âge habituel d'entrée en ponte ( 6 mois), provoque l'apparition de glandes capables de stocker des spermatozoïdes 11 jours seulement après l'injection (Lorenz et al, 1966). L'injection de $17 \beta$-œstradiol à des dindes âgées à 1, 6 ou 8 semaines provoque les mêmes effets que ceux observés chez les poules (Pescatore et Marquez, 1977), alors que l'entrée en ponte à lieu vers l'âge de 8 mois dans cette espèce.

Chez les femelles en ponte «les glandes utéro-vaginales sont des invaginations tubulaires discrètes et non branchées sur l'épithélium vaginal» (Bakst, 1987). Elles sont constituées d'une seule couche de cellules entourant une lumière centrale (fig 3). D'après Gilbert et al (1968), ces cellules sont de type non cilié à l'exception de celles qui se situent dans la partie la plus haute de la glande, près de la lumière oviducale. Ces dernières cellules, de forme pyramidale, ont leur noyau en position apicale, alors qu'il est en position basale dans les cellules non ciliées (Hodges, 1974). Ces glandes ont, chez la poule, une longueur moyenne de 400 à $600 \mu \mathrm{m}$ (mais certaines d'entre elles peuvent atteindre $2000 \mu \mathrm{m}$ ) et un diamètre extérieur de 40 à $80 \mu \mathrm{m}$, alors que le diamètre de la lumière ne dépasse pas 15-20 $\mu \mathrm{m}$ (Mero et Ogasawara, 1970). Elles sont entourées par un réseau capillaire complexe relié aux sys-

Fig 2. a) Muqueuse utéro-vaginale et spermatozoïdes (Spz) en cours de migration vers les glandes de stockage. b) Quelques spermatozoïdes commençant à pénétrer dans le col (C) de la glande visible sur la photo au MEB (avec l'aimable autorisation du Dr M Bakst). 


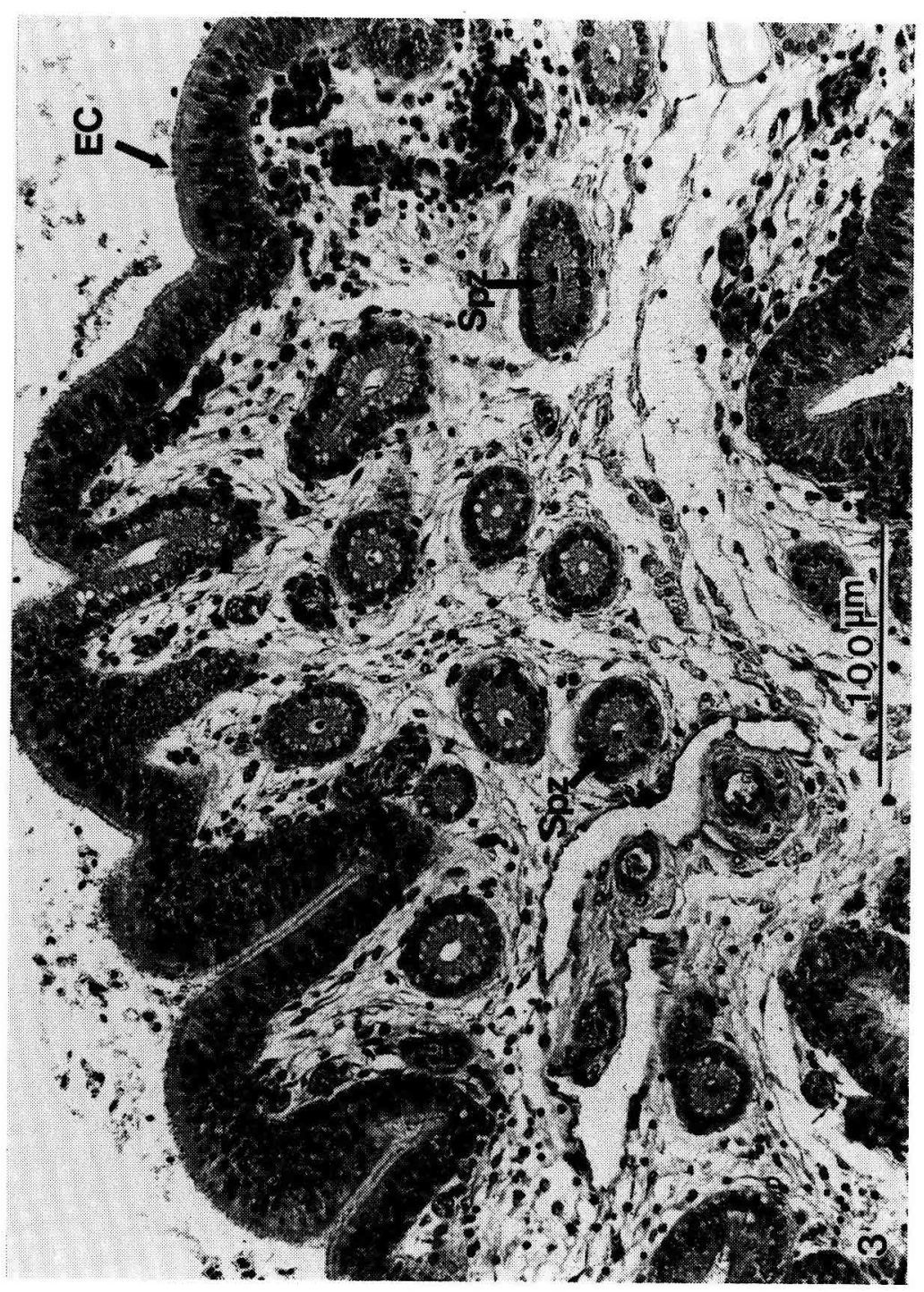

袅品

os

홇

등

으

동 동

员

을

8 . क

흥

娄 $\Sigma$

훙:응

옹 긍

롱

흥 융

뚜욱 윰 원 즈 包 응 ब. 프응 क के 둥 \% 응흥

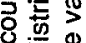
둥 Ф : สำ 袲哭 이응 票 क 능 $\stackrel{0}{\circ}$ 동 어을 क 윤 
tèmes veineux et artériel de l'épithélium adjacent. Ces glandes ne sont pas innervées (Gilbert et al, 1968) et ne présentent pas d'activité contractile qui puisse être associée à une action quelconque de remplissage ou de vidange (Van Krey et al, 1966).

Au plan ultrastructural, les cellules glandulaires sont constituées d'un noyau unique, arrondi (diamètre : 4-8 $\mu \mathrm{m}$ ) situé en position basale (Burke et al, 1972). Le cytoplasme contient un appareil de Golgi classique, ainsi que des mitochondries, soit réparties au hasard dans la région supranucléaire, soit orientées selon le grand axe de la cellule dans la région infranucléaire (Tingari et Lake, 1973). Ces derniers auteurs signalent aussi de nombreuses vacuoles ainsi que des filaments fins non striés qui ressemblent à des tonofibrilles et qui sont orientés selon le grand axe de la cellule. L'examen de la partie luminale des cellules non ciliées révèle des microvillosités identiques en taille et en forme, ainsi qu'un plateau strié. Dans la partie supérieure des glandes, les cils alternent avec des microvillosités simples ou ramifiées. Ils sont organisés selon la structure classique ( 9 doublets périphériques et un doublet central) avec une hampe ciliaire, un corps basal et des radicules striées en croix.

\section{Histochimie}

Une étude des principales substances retrouvées dans les cellules des glandes utéro-vaginales a récemment été publiée par Bakst (1987). Brièvement, les lipides sont en quantités variables selon le stade fonctionnel de l'oviducte (Fujii, 1963). Chez des poules en pause, ils occupent essentiellement la région supranucléaire, alors que chez des poules ayant pondu ils peuvent être distribués dans tout le cyto- plasme. Ces lipides seraient, soit des esters de cholestérol (Fujii, 1963), soit des lipides associés à d'autres substances (Gilbert et al, 1968). En de rares circonstances, ils peuvent envahir la lumière glandulaire sous la forme d'une grosse goutte. Leur fonction réelle est à ce jour inconnue : selon Renden et al (1981), ils pourraient participer au maintien de l'intégrité structurale des spermatozoïdes, alors que pour Bakst (1987) ils seraient utilisés comme substrat métabolique. La présence de lipides est constante dans les espèces étudiées : canard (Pal, 1977); dinde (Schuppin et al, 1984). A l'inverse, du glycogène a été retrouvé chez la poule (Lorenz et al, 1967; Gilbert et al, 1968) alors que, chez la dinde, il semble inexistant (Bradley, 1982; Bakst, 1987) ou présent à l'état de traces (Schuppin et al, 1984). Notons enfin qu'aucune variation histochimique du contenu des glandes utéro-vaginales au cours de la période de ponte n'a pu être établie (Bradley, 1982; Schuppin et al, 1984) ce qui rend peu probable l'existence d'un cycle secrétoire saisonnier.

\section{Relations spermatozoïdes-glandes de stockage : aspects morphologiques}

Pour Fujii et Tamura (1963), les spermatozoïdes entreraient en contact avec les cellules glandulaires au niveau des espaces intercellulaires existant dans la région luminale des glandes. A cet endroit, ils forment des paquets denses difficiles à dissocier. Mais seuls les spermatozoïdes contenus dans ces agglutinats ont une structure normale : ceux qui sont libres dans la lumière ont en général une lésion morphologique évidente (disparition de l'acrosome ou du flagelle). Selon Van Krey et al (1967), les spermatozoïdes stockés dans la région utéro-vaginale sont retrouvés dans la ré- 
gion aveugle des glandes, profondément enfouis dans les microvillosités bordant la région luminale des cellules qui les composent. Ces microvillosités sont à la fois plus longues et plus denses dans cette région que dans la partie supérieure de la glande. Pour Van Krey et al (1967), elles pourraient avoir une double action sur les spermatozoïdes, à la fois en leur fournissant des substances nutritives (sous forme de granules sécrétoires) et en absorbant les déchets métaboliques accumulés dans les glandes. Mais pour Burke et al (1972), les spermatozoïdes ne seraient en contact avec les microvillosités que lorsqu'ils sont stockés en très grand nombre. Dans la plupart des coupes examinées, ces auteurs ne constatent qu'une tendance des têtes de spermatozoïdes à se disposer en périphérie de la lumière (contre les microvillosités), alors que les flagelles sont plutôt rassemblés dans les centres de celleci. Tingari et Lake (1973) notent que les spermatozoïdes présents dans la lumière des glandes sont orientés de façon caractéristique : ils sont parallèles entre eux et au grande axe de la glande, leur tête étant tournée vers l'extrémité aveugle de celleci. Les têtes des spermatozoïdes semblent accolées les unes aux autres "de telle sorte, soulignent-ils, que la coupe transversale d'une glande en contenant laisse apparaître des fragments cellulaires identiques entre spermatozoïdes adjacents». La membrane cytoplasmique enveloppant la tête semble, dans certains cas, être en association intime avec les microvillosités de l'épithélium glandulaire et, dans d'autres, avec des spermatozoïdes adjacents. Pour Van Krey et al (1981), la disposition "tête contre tête" des spermatozoïdes dans les glandes utéro-vaginales constitue un phénomène d'agglutination qui ne se manifeste qu'avec des spermatozoïdes ayant conservé leur intégrité membranaire, et disparaît peu à peu au cours de leur vieillissement. Cette capacité d'agglutina- tion serait le mécanisme principal permettant le stockage prolongé des spermatozoïdes dans les glandes utéro-vaginales, ceux qui restent libres étant progressivement éliminés vers la lumière de l'oviducte. Schuppin et al (1984) observent chez la dinde les mêmes formes d'agglutination que celles décrites par Tingari et Lake puis Van Krey et al (1981) chez la poule. Chez les dindes infertiles, les glandes utérovaginales sont pratiquement vides de spermatozoïdes bien qu'aucune différence ultrastructurale n'apparaisse entre ces glandes et celles, normalement remplies, des femelles fertiles (Schuppin et al, 1984). Cette dernière observation conforte l'hypothèse selon laquelle l'absence de spermatozoïdes dans les glandes de la jonction utérovaginale chez des femelles infertiles pourrait plus être due à un facteur empêchant la remontée des gamètes mâles au niveau du vagin qu'à l'absence d'une sécrétion nécessaire à leur survie prolongée.

\section{RELATIONS SPERMATOZOIIDES- GLANDES DE STOCKAGE : ASPECTS FONCTIONNELS}

Les mécanismes responsables de l'entrée puis du stockage des spermatozoïdes dans les glandes spécialisées de l'oviducte restent à ce jour inconnus (Zavaleta et Ogasawara, 1987), mais plusieurs informations récentes laissent entrevoir des progrès rapides sur ce point. Ces informations impliquent directement ou indirectement la nature chimique de l'enveloppe cellulaire externe des spermatozoïdes (glycocalyx) et les changements qu'elle subit lors du transit des gamètes mâles dans les voies déférentes (canaux de Wolff). Dans cette région, les cellules épithéliales sécrètent plusieurs protéines qui se déposent à la surface des spermatozoïdes (Esponda et 
Bedford, 1985; Morris et al, 1987). Ces protéines pourraient être impliquées dans le transport et/ou la survie prolongée des gamètes mâles dans les voies génitales femelles. L'enveloppe protéique ainsi constituée reste "attachée" aux spermatozoïdes lors de leur stockage utéro-vaginal ou infundibulaire (Morris et al, 1987). Or, Van Krey et al (1981) ont, d'une part, confirmé les observations ultrastructurales de Tingari et Lake (1973) relatives à l'agglutination des spermatozoïdes au cours de leur stockage in vivo et, d'autre part, avancé l'hypothèse selon laquelle cette agglutination constitue le mécanisme responsable de leur stockage prolongé. En partant de ces remarques, plusieurs auteurs se sont intéressés au rôle de l'enveloppe protéique externe qui entoure les spermatozoïdes, en traitant ceux-ci par de la neuraminidase, une enzyme qui détache l'acide sialique des glycoprotéines. Après un tel traitement, Froman et Thurston (1984) observent que les capacités de fécondation des spermatozoïdes sont altérées bien que leur motilité soit maintenue. Pour Lake et Ravie (1988), la suppression des résidus d'acide sialique accolés aux protéines membranaires empêche les glandes utérovaginales de reconnaître les spermatozoïdes, interdisant ainsi le stockage de ceux-ci après insémination intravaginale. Cependant, Froman et Engel (1989) ont établi que le traitement à la neuraminidase, qui diminue le taux de fécondation après insémination intravaginale, n'altère pas ce taux lorsque les spermatozoïdes sont déposés par voie intramagnale (favorisant le stockage infundibulaire). Ces derniers auteurs concluent que l'absence d'acide sialique sur la couche glycoprotéique externe des spermatozoïdes ne change pas leur aptitude à reconnaître les ovocytes, mais supprime plutôt leur capacité d'agglutination dans les glandes de la jonction utéro-vaginale.
Quel est le rôle réel de l'acide sialique présent à la surface des glycoprotéines membranaires ? A-t-il, comme Lake et Ravie (1988) l'avancent, une action sur la reconnaissance des spermatozoïdes par les cellules des glandes utéro-vaginales ou bien favorise-t-il l'agglutination qui semble nécessaire à un stockage prolongé ? Si cette seconde hypothèse était retenue, il faudrait alors se demander pourquoi des spermatozoïdes traités à la neuraminidase persistent longtemps dans l'infundibulum, alors que, dans les mêmes conditions, un stockage utéro-vaginal est impossible (Froman et Engel, 1989). Le mécanisme d'agglutination est-il différent selon le site de stockage ? Le processus d'évacuation des spermatozoïdes, par «effeuillage» progressif proposé par Van Krey et al (1981), pour la région utéro-vaginale, est-il remis en cause au niveau infundibulaire?

\section{CONCLUSION}

S'il est maintenant établi que chez les oiseaux femelles, les spermatozoïdes sont stockés dans la jonction utéro-vaginale, de nombreuses questions concernant les relations entre ces cellules et celles qui appartiennent à leur zone de stockage restent sans réponse. Ainsi, l'existence d'un cycle sécrétoire des cellules composant le corps des glandes utéro-vaginales reste possible mais, selon Schuppin et al (1984), puis Bakst (1987), l'absence d'organites cytoplasmiques associés à la synthèse protéique suggère plutôt que ces cellules ont une fonction d'absorption qui, par élimination des substances catabolisées par des spermatozoïdes, maintiendrait durablement un milieu compatible avec leur survie. Le rôle de pompe à déchets pourrait alors être assuré par les nombreuses microvillosités bordant la lumière des glandes. 
Les raisons pour lesquelles les spermatozoïdes sont stockés durablement dans les glandes utéro-vaginales n'ont pas non plus été élucidées, mais les observations récentes que nous venons d'évoquer laissent penser que des réactions immunitaires locales font intervenir soit un processus de reconnaissance des spermatozoïdes par les cellules qui tapissent l'entrée des glandes utérovaginales, soit leur agglutination pendant leur séjour dans ces glandes. Une estimation précise de la vitesse de stockage des spermatozoïdes, avec ou sans altération des récepteurs présents à la surface de leur glycocalyx, devrait fournir des éléments de réponse à cette importante question.

\section{REMERCIEMENTS}

L'auteur est tout particulièrement reconnaissant au Dr M Bakst (Avian Physiology Laboratory, USDA, Beltsville, USA) qui a accepté de fournir les documents photographiques reproduits dans cet article. II remercie également Mmes C Lessire, O Barbereau ainsi que Mlle M Plouzeau, INRA, Station de recherches avicoles, Nouzilly, 37380 Monnaie, France) pour la qualité du travail de présentation et la recherche des documents bibliographiques.

\section{RÉFÉRENCES}

Allen TE, Grigg GW (1957) Sperm transport in the fowl. Aust J Agric Res 8, 788-799

Bakst MR (1987) Anatomical basis of sperm storage in the avian oviduct. Scanning Electron Microscopy 1, 1257-1266

Bakst MR, Bird DM (1987) Localization of oviducal sperm-storage tubules in the American kestrel (Falco sparverius). Auk 104, 321-324

Bobr LW, Lorenz FW, Ogasawara FK (1964) Distribution of spermatozoa in the oviduct and fertility in domestic birds. II. Transport of Spermatozoa in the fowl oviduct. $J$ Reprod Fertil 8, 49-58
Bradley FAM (1982) The ultrastructure and function of the primary sperm-storage sites in the turkey hen, as affected by season and insemination. PhD Thesis, University of Davis, Diss Abstr 43, 3148B

Bray OE, Kennelly JJ, Guarino JL (1975) Fertility in eggs produced on territories of vasectomized red-winged blackbirds. Wilson Bull 87, 187-195

Burke WH, Ogasawara FX, Fuqua CL (1972) A study of the ultrastructure of the utero-vaginal sperm storage glands of the hen, Gallus domesticus, in relation of a mechanism for the release of spermatozoa. J Reprod Fertil 29, 29-36

Degen AA, Hawes RO (1972) Fertility in the domestic hen following the surgical removal of the utero-vaginal junction. Poult Sci 51, 464470

Esponda P, Bedford JM (1985) Surface of the rooster spermatozoon changes in passing through the Wolffian duct. J Exp Zool 234, 441-449

Elder WH, Weller MW (1954) Duration of fertility in the domestic Mallard hen after isolation from the drake. J Wild Manage 18, 495-502

Frieb AE, Sinowarz F, Wrobel KH (1978) The utero-vaginal sperm host glands of the quail (Coturnix coturnix japonica). Cell Tissue Res, 115-119

Froman DP, Thurston RJ (1984) Decreased fertility resulting from treatment of fowl spermatozoa with neuraminidase or phospholipase C. Poult Sci 63, 2479-2482

Froman DP, Engel HN Jr (1989) Alteration of the spermatozoal glycocalyx and its effect on duration of fertility in the fowl (Gallus domesticus). Biol Reprod 40, 615-621

Fronda FM, Zialcital P, Dalisay AM (1940) The fertility of the duck egg. Philipp Agric 29, 111123

Fujii S (1963) Histological and histochemical studies on the oviduct of the domestic fowl with special reference to the region of uterovaginal junction. Arch Histol JPN 23, 447-459

Fujii S, Tamura T (1963) Location of sperms in the oviduct of domestic fowl with special reference to storage of sperms in the vaginal gland. J Fac Fish Anim Husb Hiroshima Univ $5,145-163$ 
Gilbert AB, Reynolds ME, Lorenz FW (1968) Distribution of spermatozoa in the oviduct and fertility in domestic birds. V. Histochemistry of the utero-vaginal sperm host glands of the domestic hen. $J$ Reprod Fertil 16, 433444

Hale EB (1955) Duration of fertility and hatchability following natural matings in turkeys. Poult Sci 34, 228-233

Hatch SA (1983) Mechanism and ecological significance of sperm storage in the Northern Fulmar with reference to its occurence in other birds. Auk 100, 593-600

Hodges RD (1974) The female reproductive system. In: The Histology of the fowl (Hodges RD, ed), Academic Press, London, 6, 387

Johnson AS (1954) Artificial insemination and the duration of fertility of geese. Poult Sci 33, 638-640

Kinney T, Burger RE (1960) A technique for the insemination of geese. Poult Sci 39, 230-232

Koyanagi F, Nishiyama $H$ (1981) Disintegration of spermatozoa in the infundibular sperm host glands of the fowl. Cell Tissue Res 214, 81-87

Lake PE (1967) The maintenance of spermatozoa in the oviduct of domestic fowl. In: Reproduction in the Female Mammal. Proc 13th Easter School in Agricultural Science, Univ Nottingham, 1966 (GE Fanning, EC Amoroso, eds), Butterworths, London, 254-266

Lake PE (1975) Gamete production and the fertile period with particular reference to domesticated birds. Symp Zool Soc Lond 35, 225 244

Lake PE, Ravie O (1988) Effect of trypsin and neuramidinase on fowl spermatozoa and their retention in the oviduct. Proc XVIlth World's Poultry cong, Nagoya, 594-595

Lodge JR, Fechheimer NŚ, Jaap RG (1971) The relationship of in vivo sperm storage interval to fertility and embryonic survival in the chicken. Biol Reprod 5, 252-257

Lorenz FW (1950) Onset and duration of fertility in turkeys. Poult Sci 29, 20-26

Lorenz FW, Ogasawara FX (1968) Distribution of spermatozoa in the oviduct and fertility in domestic birds. VI. The relations of fertility and embryo normality with site of experimental insemination. J Reprod Fertil 16, 445-455
Lorenz FW, Reynolds ME, Howard DL (1966) Endocrine control of development of sperm host glands in the chick. Fed Proc 25, 190

Lorenz FW, Reynolds ME, Guilbert AB (1967) Secretory activity of the sperm host glands of the domestic fowl. Physiologist 10, 236

McCartney MG (1951) The physiology of reproduction in turkeys. 2. Degree and duration of fertility and hatchability in broody and nonbroody pullets. Poult Sci 30, 663-667

Martinet L, Raynaud F (1972) Mécanisme possible de la superfétation chez la hase. $C R$ Acad Sci Paris, Serie D 274, 2683-2686

Mero KN, Ogasawara FX (1970) Dimensions of utero-vaginal sperm storage tubules of the chicken and their possible significance in sperm release. Poult Sci 49, $1304-1308$

Morris SA, Howarth B Jr, Grim JW, Rodriguez de Cordoba $S$, Esponda $P$, Bedford JM (1987) Specificity of sperm-binding Wolffian duct proteins in the rooster and their persistence on spermatozoa in the female host glands. J Exp Zool 242, 189-198

Nicolaïdes $C$ (1934) Fertility studies in poultry. Poult Sci 13, 178-183

Ogasawara FX, Lorenz FL, Bobr LW (1966) Distribution of spermatozoa in the oviduct and fertility in domestic birds. III. Intra-uterine insemination of semen from low-fecundity cocks. J Reprod Fertil 11, 33-41

Ogasawara FX, Fuqua CL (1972) The vital importance of the utero-vaginal sperm host glands for the turkey. Poult Sci 51, $1035-$ 1039

Pal D (1977) Histochemistry of the utero-vaginal junction with special reference to the sperm host glands in the oviduct of the domestic duck. Folia Histochem Cytochem 15, 235242

Pescatore AJ, Marquez BJ (1977) Sperm storage tubule development in immature turkeys injected with female sex steroïds. Poult Sci 56, 1746-1 747

Prasad S (1967) Histological location and role of utero-vaginal glands in storage and release of spermatozoa in chickens and turkeys. PhD Thesis, University of Missouri, Columbia. Diss Abst 28, 2114 B

Renden JA, Moy EB, Benoff FH (1981) Histochemistry of utero-vaginal sperm host glands 
in japanese quail (Coturnix coturnix Japanica) with reference to the period of ovoposition. Poult Sci 60, 2529-2535

Richardson KC (1935) The secretory phenomena of the oviduct of the fow1, including the process of shell formation examined by the microincineration technique. Philos Trans $R$ Soc Lond B Biol Sci 225, 149-195

Romanoff AL (1960) Fertilization and fertility. In: The Avian Embryo, Macmillan, New York, 75-111

Saeki $Y$, Tanabe $Y$, Katsyraci $T$, Miyagi $M$ (1963) Studies on the advance and distribution of cock sperm in the oviduct with special reference to the tracing method with $32 \mathrm{P}$ labelled sperm. Bull Natl Inst Anim Ind 3, 9198

Sauveur B (1979) Conservation prolongée et transport des spermatozoïdes dans les voies génitales des oiseaux. In : Oviducte et fertilité (I Brosens et al, eds) Masson, Paris, 6586

Schindler H, Ben-David E, Hurwitz S, Dempenich $O$ (1967) The relation of spermatozoa to the glandular tissue in the storage sites in the hen oviduct. Poult Sci 46, 1462-1471

Schuppin GT, Van Krey HP, Denbow B, Bakst MR (1984) Ultrastructural analyses of uterovaginal sperm storage glands in fertile and infertile turkey breeder hens. Poult Sci 63, $1872-1882$

Sittmann K, Abplanalp H (1965) Duration and recovery of fertility in japanese quail (Coturnix coturnix japonica). Br Poult Sci 5, 245250

Takeda A (1964) Behavior of spermatozoa in the female genital tract of the hen. I. Presence of spermatozoa in the oviduct. $J p n$ Poult Sci 1, 19-31

Thibault C, Levasseur MC (1973) Conservation prolongée des spermatozoïdes dans les voies génitales femelles des vertébrés. Ann Biol Anim Biochim Biophys 13, 267-284

Tingari MD, Lake PE (1973) Ultrastructural studies on the utero-vaginal sperm host glands of the domestic hen, Gallus domesticus. J Reprod Fertil 34, 423-431

Van Drimmelen GC (1946) Sperm nests in the oviduct of the domestic hen. JS Afr Vet Med Assoc 17, 42-52

Van Krey HP (1965) Storage and transport of spermatozoa within the oviduct of the domestic fow1. PhD, University of Davis. Diss Abstr 28, 2841

Van Krey HP, Ogasawara FX, Lorenz FW (1964) Relative storage potential of the uterovaginal and infundibular sperm glands. Poult Sci 43,1373

Van Krey HP, Ogasawara FX, Lorenz FW (1966) Distribution of spermatozoa in the oviduct and fertility in domestic birds. IV. Fertility of spermatozoa from infundibular and uterovaginal glands. $J$ Reprod Fertil 11, 257-262

Van Krey HP, Ogasawara FX, Pangborn J (1967) Light and electron microscopic studies of possible sperm gland emptying mechanisms. Poult Sci 46, 69-78

Van Krey HP, Balander RJ, Compton MM (1981) Storage and evacuation of spermatozoa from the utero-vaginal sperm host glands in domestic fowl. Poult Sci 60, 871-877

Verma OP, Cherms FL (1964) Observations on the oviduct of turkeys. Avian Dis 8, 19-26

Wimsatt WA (1942) Survival of spermatozoa in the female reproductive tract of the bat. Anat $\operatorname{Rec}$ 83, 299-307

Zavaleta D, Ogasawara FX (1987) A review of the mechanism of the relase of spermatozoa from storage tubules in the fowl and turkey oviduct. World's Poult Sci J 43 (2), 132-139 